\title{
Improving product quality in pharmaceutical ball milling
}

Ball milling is used in the pharmaceutical industry to material such as pharmaceutical granules. Dr Alexander Krok and Dr Sandra Lenihan from Mun Technological University, computationl approach to achieve optimal, energy-efficient resulting in the production of high-quality particulate material (i.e. granules) of homogeneous shape and size. size reduction in ball milling

all milling is a technique that is each factor affects the average size of the Uutilised by the pharmaceutical industry for granular size reduction of drugs or excipients. There are many astens to to have a specific surace arear might be a process requirement that the from throughout the whole batch. This is because the shape, size and the surface area-to-volume ratio of the particles are parameters that affect critical properties of the material, such as its solubility or its reactivity in the chemical processes for which it is employed. Variables such as the rotation speed of the drum and the physical properties of balls and milling material have the most significa influence on the final quality of the product. It is also necessary to consider the optimum filling level of both milling material and balls. In order to achieve costs, it is important yede wh minimal

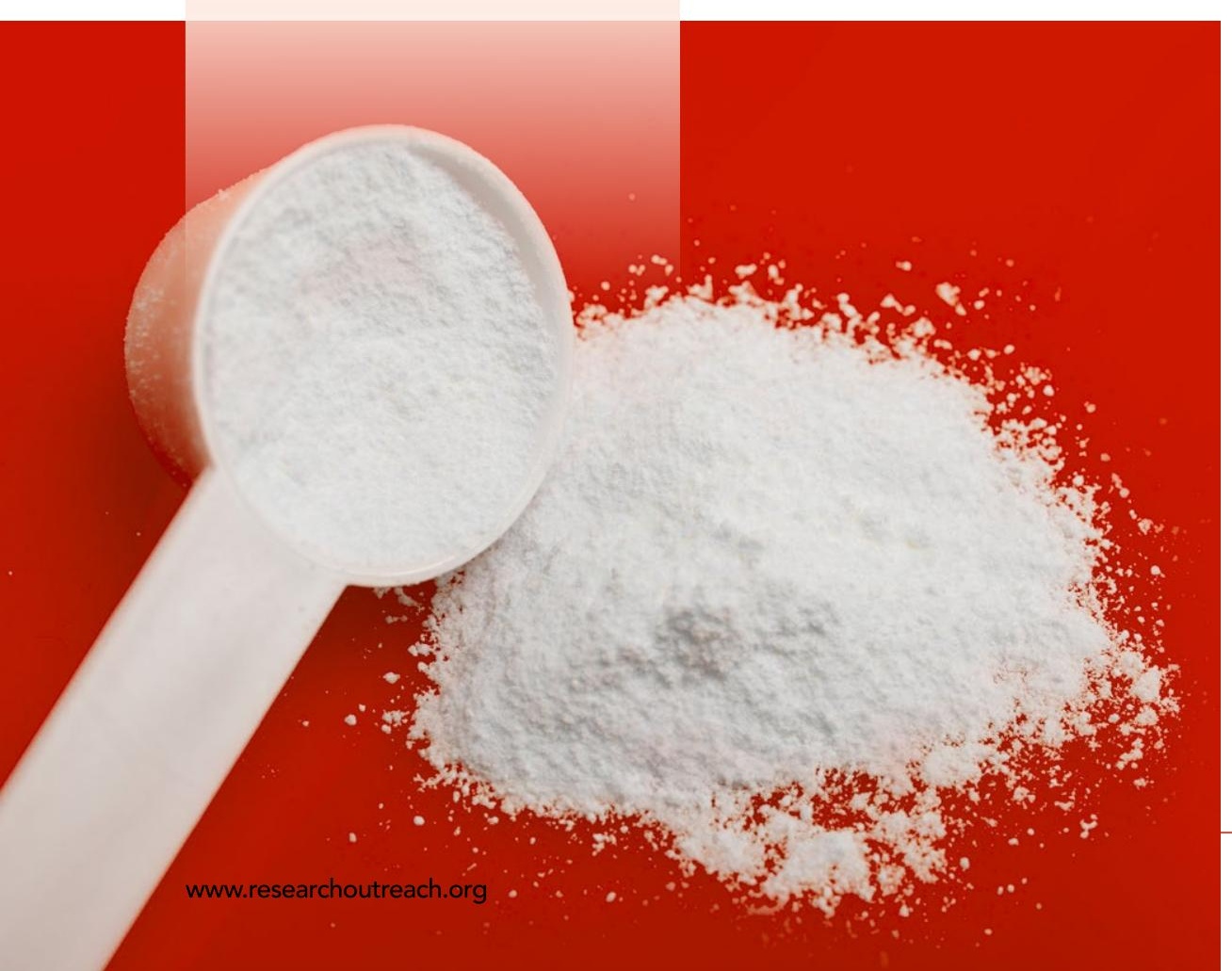

particulate fragments in the final product. Traditional approaches often fail to predict the mechanisms of fragmentation

Dr Alexander Krok and Dr Sandra University, Ireland, have developed advanced computational models to granules with non-uniform shape and pharmaceutical industry. Drs Krok and Lenihan aim to study and validate the effectiveness of a computationa method known as the discrete element method (DEM), instead of a technique used in previous research where the breaking behaviour was estimated through the impact energy for fracturing.

\section{FFFECT OF MILLING SPEED ON}

\section{GRANULE FRAGMENTATION}

The optimisation of energy

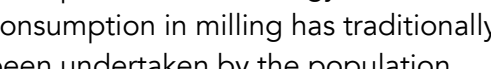

balance modelling (PBM) approach.

Some of the parameters used in PBM

calculations must be determined

experimentally, making this entire

process time consuming. As a

consequence, DEM has been growing

in popularity among researchers in

the field, providing significant insights

into the operation of pharmaceutical

granule milling.

Drs Krok and Lenihan investigated the fragmentation of bonded particles into DEM granules of bilferent shape and size during the ball milling process
and have reported their findings in a Lenihan, from Munster Technological different size in ball milling for the of ball collisions as the driving force
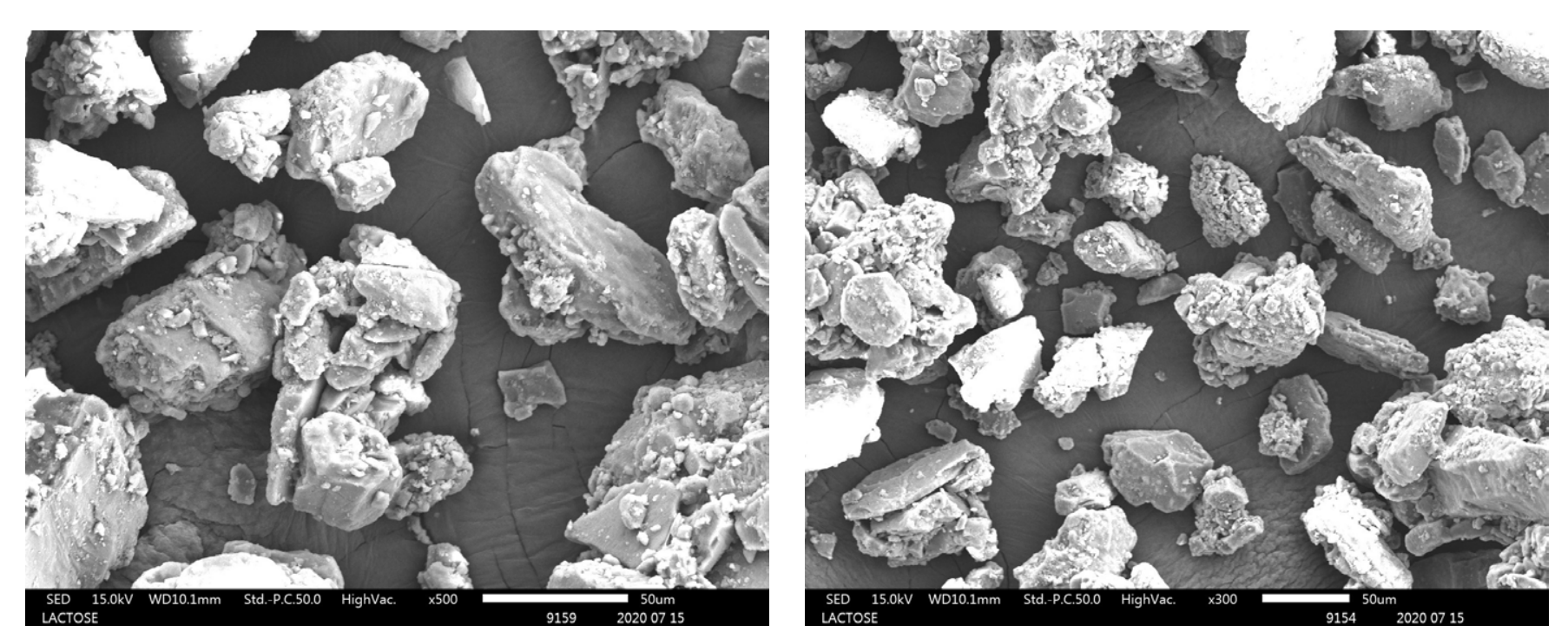

Figure 1. Scanning electron micrographs of granule lactose with magnitudes a) $\times 500$ and b) x1000.

recently published paper. The study The predicted shapes of the different explores the effect of the rotation granules were sorted into three main breakage behaviour of DEM granules a calculated sphericity between 0.75 all milling process. One of the conclusions of the study was and 0.93 , and roundness between

granules where sphericity varied vetween 0.6 and 0.85 , and round 0.25 and 0.6 .

\section{CONFIGURATION}

\section{In the pilot study,}

between the balls and the product The shape, size and the surface area conceived as a steel granules increased to volume ratio of the particles are cylindrical shell with of the material, such as its solubility.

was fitted with eight ill power also speed. The $\quad 1.2 \mathrm{~mm}$ in height and $0.8 \mathrm{~mm}$ in width. speed. The relationship between the 'blocky' granules had narrower The milling action was provided by ten number of fragments produced and the ranges of sphericity and roundness, steel balls with a density of $7800 \mathrm{~kg} / \mathrm{m}$ specifically between 0.65 and 0.92 for The number of balls to be employ

\section{PREDICTING THE BREAKAGE} PATTERN OF THE GRANULES The material used for the study was lactose, an excipient commonly used in the pharmaceutical industry. The granule shape was described taking into account the parameters of sphericity and roundness. In their paper, the researchers defined sphericity as the ratio between the outer and internal diameters of the granules. Roundness indicates the sharpness of particle corners and was defined as the ratio of the average radius of curvature of the corners of a particle to the radius of the maximum inscribed circle. The researchers used a system of equations, so that the granule shape can be determined by the calculated sphericity and between 0.25 and 0.6 was carefully optimised, as it was Peters of sphericity and roundness.

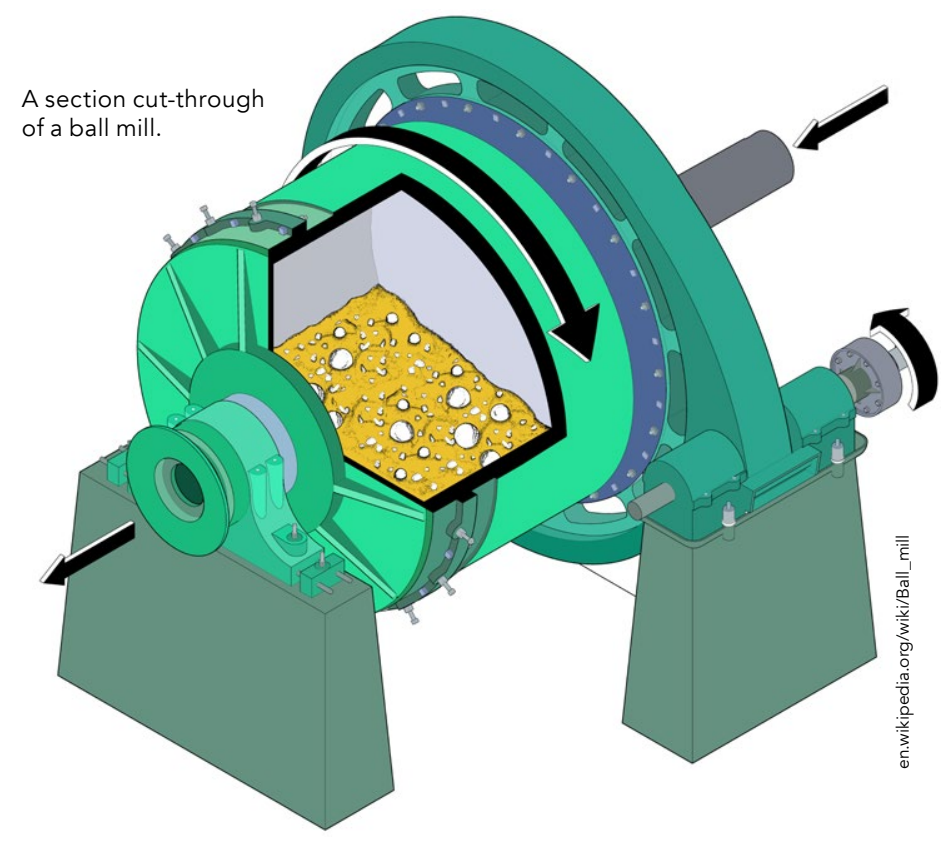




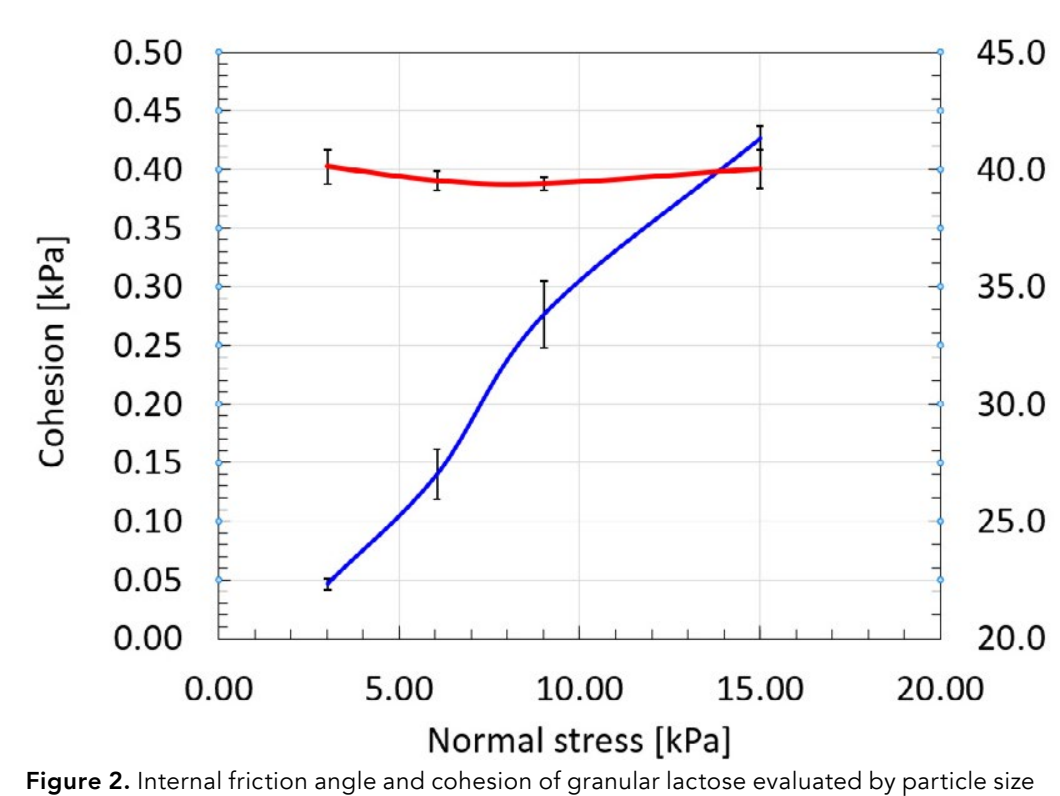

Figure 2. Internal friction an
analyser and $\mathrm{FT}$ rheometer.

is more energy efficient as it produces consumption. For their calces power the researchers conceived the drum as a counter-clockwise rotating cylinder consisting of four individual zones: an

CONCLUSIONS OF THE STUDY
Drs Krok and Lenihan, along with their collaborators, conducted a systematic simulation study on the process of

pharmaceutical ball milling. The aim of their study was to investigate the

DEM approach a strong correlation between the impact speed and the - with previous data published in the scientific literature. A rotation speed of $190 \mathrm{rpm}$ (revolutions per minute) was the most effective milling speed in the study and led to optimal reduction in particle size. Increasing the rotation speed above this value was counterproductive and was characterised by centrifugal motion, which negatively impacted the fragmentation process. On the other hand, lower rotation speeds caused a rolling-cascading particle motion, which was not adequate for effective ball milling. However, the researchers point out that while increasing the rotation speed up to $190 \mathrm{rpm}$ produced the most effective collisions between the increased the calculated power consumption of the milling process.

The computational method developed Drs Krok and the top left quarter A rotation speed of $190 \mathrm{rpm}$ was the most product, while section, a'dead effective milling speed in the study and costs associated zone' at the top of the bottom right led to optimal reduction in particle size. Reducing the quarter, an 'abrasion zone', just below the 'dead zone' in the bottom right quarter and a 'high impact zone' in the bottom left quarter of the the size of granular lactose particles. cylinder's section, where the mill balls simulation of therm the numerical collide effectively. granules and ensuring that they are homogeneous with regards to their shape will result in the optimum physical and chemical properties within the desired pharmacolosican

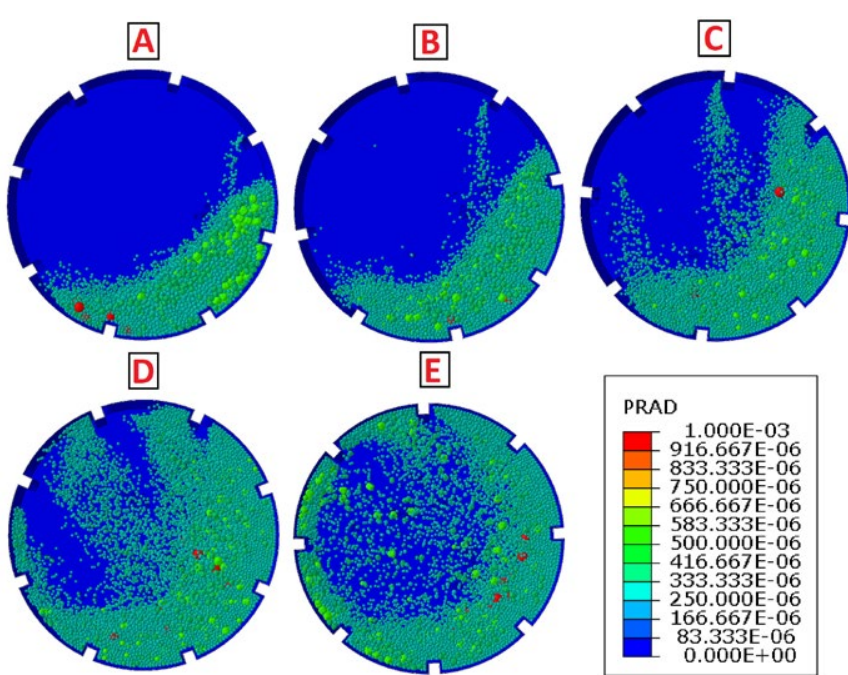

Figure 3. The size distribution of granules in the drum during ball milling
with varying rotation speed: a) 47 rpm; b) 95 rpm; c) 143 rpm; d) 190 rpm with varying rotator
and e) 238 rpm.

\section{BEGINNING}

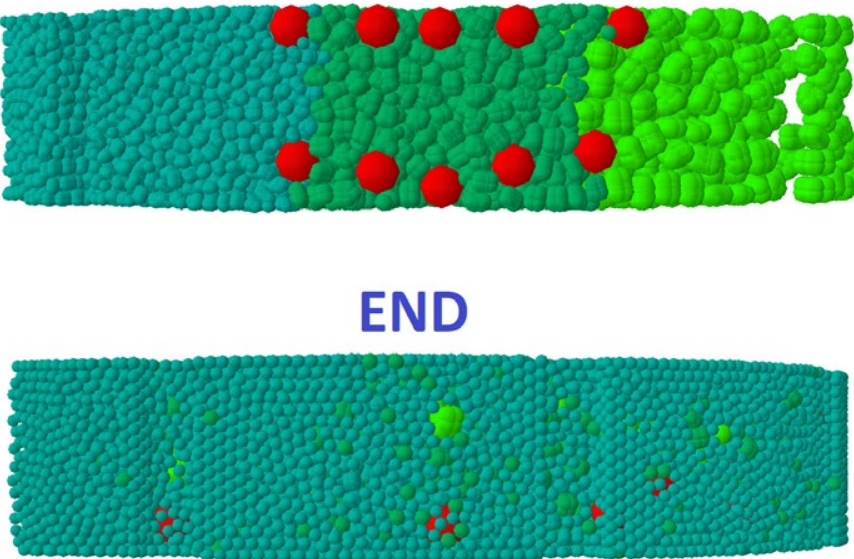

Figure 4. Typical perspective from the base of the drum side at the
beginning and the end of the ball milling ocess

\section{Behind the Research}

E: alexander.krok@cit.ie E: sandra.lenihan@cit.ie T: +353876053311 W: https://www.mtu.ie W: https://www.cit.ie

\section{Research Objectives}

Dr Krok and Dr Lenihan are adopting computational modelling to investigate the mechanism of particle size reduction in ball milling.

\section{Detail}

\section{Address}

Muster Technological University

(astive, Bishopstown

Cork, T12 p92

Bio

Dr Alexander Krok graduated with a BSc degree

in Organic Chemistry and MSc degree in Chemica

Engineering at the Faculty of Chemical and Food

Technology (FCHPT-STU, Slovakia). He completed a PhD in Chemical and Process Engineering at the Faculty of Mechanical Engineering, Slovak University of Technology (SjF-STU, Slovakia) in 2011. Currently, he is a Lecturer at Munster Technological University (MTU, Ireland). Dr Krok has significant experience in computational modelling with a deep knowledge of theoretical aspects of chemical and process engineering, powder technology and in pharmaceutical engineering processes.

Dr Sandra Lenihan graduated with a degree in Industria Chemistry from the University of Limerick (UL, Ireland) Astellas Subsequen Dr Lenis Physical Chemistry at UL She is a cecturer in Chemical and Biopharmaceutical Engineering and active applied researcher in MTU. Her interests include industriatacademic collaborations in the areas of pharmaceutica formulations, with particular focus on solid dosage processing, physical characterisation, and chemical and biopharmaceutical engineering.

Funding

This work was supported by the Career-FIT Fellowships, funded through European Union's Horizon2020 research and innovation programme under the Marie Skłodowsk Curie grant agreement No. 713654 .

Collaborators - Peter Peciar - Kieran Coffey ENTERPRISE
IRELAND

\section{References}

Krok, A., Peciar, P., Coffey, K., Bryan, K., and Lenihan, S. (2021). A combination of Density-Based Clustering method and DEM to numerically investigate the breakage of bonded pharmaceutical granules in the ball milling process. Particuology, 58, 153-168. Available at: https://doi. org/10.1016/j.partic.2021.03.008

Metzger, M.J. and Glasser, B.J. (2012). Numerical investigation of the breakage of bonded agglomerates during impact. org/10.1016/jpontec 2011.10.042

\section{Personal Response}

\section{What are the next steps in your research?}

II We are actively developing high-quality innovative and drug delivery systems, with the primarily focus being in the pharma sectors. The research activities of both are using advanced methodologies to existing processing problems (granulation, coating), mainly its mechanistic modelling and in pharmaceutical engineering by utilising cutting gedge characterisation techniques and visualisation techniques to correctly interpreted and visualize the results from different
pharmaceutical units operation.

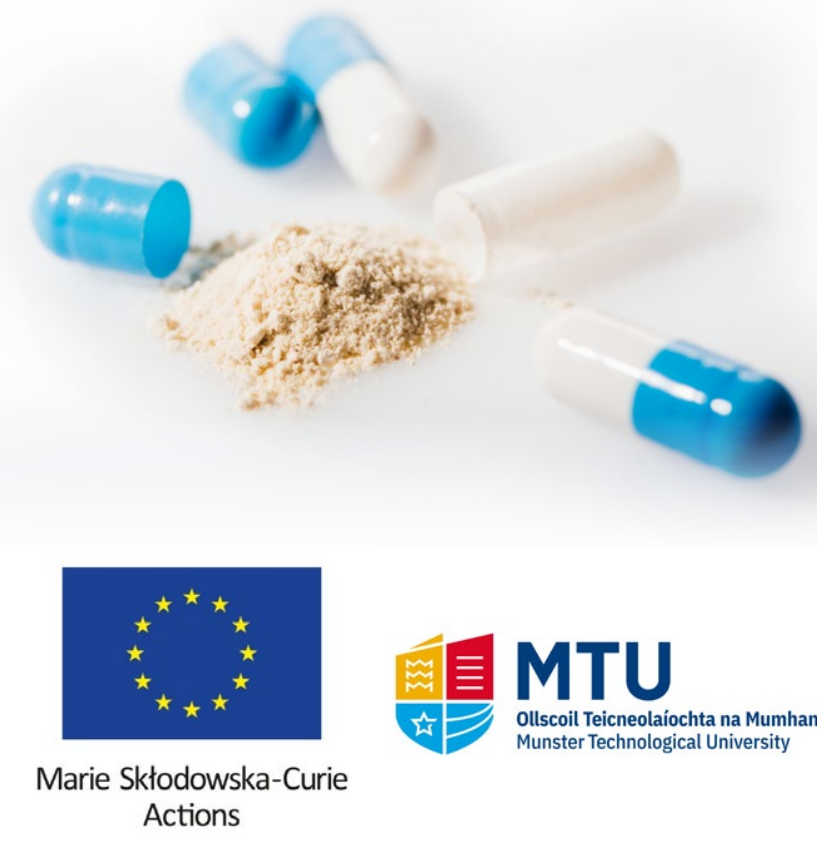

